\title{
Massively Parallel Selection of NanoCluster Beacons
}

Yu-An Kuo', Cheulhee Jung ${ }^{3}$, Yu-An Chen ${ }^{1}$, Hung-Che Kuo ${ }^{2,4}$, Oliver S. Zhao', Trung D. Nguyen', James R. Rybarski ${ }^{2,4}$, Soonwoo Hong ${ }^{1}$, Yuan-I Chen ${ }^{1}$, Dennis C. Wylie ${ }^{6}$, John A. Hawkins ${ }^{5}$, Jada N. Walker Samuel W. Shields ${ }^{7}$, Jennifer S. Brodbelt ${ }^{7}$, Jeffrey T. Petty ${ }^{8}$, Ilya J. Finkelstein ${ }^{2,4}$, and Hsin-Chih Yeh ${ }^{1,9, *}$ ${ }^{1}$ Department of Biomedical Engineering, University of Texas at Austin, Austin, USA

${ }^{2}$ Department of Molecular Biosciences, University of Texas at Austin, Austin, USA

${ }^{3}$ Department of Biotechnology, College of Life Sciences and Biotechnology, Korea University, Seoul, Korea

${ }^{4}$ Center for Systems and Synthetic Biology, University of Texas at Austin, Austin, USA

${ }^{5}$ European Molecular Biology Laboratory (EMBL), Heidelberg, Germany

${ }^{6}$ Computational Biology and Bioinformatics, Center for Biomedical Research Support, University of Texas at Austin, Austin, USA

${ }^{7}$ Department of Chemistry, University of Texas at Austin, Austin, USA

${ }^{8}$ Department of Chemistry, Furman University, Greenville, USA

${ }^{9}$ Texas Materials Institute, University of Texas at Austin, Austin, USA

NanoCluster Beacons (NCBs) are multicolor silver nanocluster probes whose fluorescence can be activated or tuned by a proximal DNA strand called the activator. While a single-nucleotide difference in a pair of activators can lead to drastically different activation outcomes, termed the polar opposite twins (POTs), it is difficult to discover new POT-NCBs using the conventional low-throughput characterization approaches. Here we report a high-throughput selection method that takes advantage of repurposed next-generation-sequencing (NGS) chips to screen the activation fluorescence of $\sim 40,000$ activator sequences. We find the nucleobases at positions 7-12 of the 18-nucleotide-long activator are critical to creating bright NCBs and positions 4-6 and 2-4 are hotspots to generate yellow and red POTs, respectively. Based on these findings, we propose a "zipper bag model" that explains how these hotspots lead to the creation of distinct silver cluster chromophores and contribute to the difference in chromophore chemical yields. Combining high-throughput screening with machine learning algorithms, we establish a pipeline to rationally design bright and multicolor NCBs.

Activatable and multicolor fluorescent probes are indispensable tools in analytical chemistry and quantitative biology as they enable sensitive detection of analytes and diagnostic imaging of biomarkers in complex environments ${ }^{1,2}$. Whereas activatable probes have greatly simplified the assays by eliminating the need to remove unbound probes, the development of new activatable probes is largely constrained by the scarce activation mechanisms (e.g., FRET), the limited activation colors (e.g., existing FRET pairs) and the poor enhancement ratios (e.g., 10- to 60-fold for a typical molecular beacon) ${ }^{3}$. NanoCluster Beacons (NCBs) ${ }^{4}$ are a unique class of activatable probes as they provide a palette of activation colors from the same dark origin ${ }^{5}$ (not via FRET) and achieve fluorescence enhancement ratios as high as $1,500^{6}$ to 2,400 -fold $^{7}$. The core of NCB is a few-atom silver nanocluster ${ }^{8-}$ 
13 (e.g., $\mathrm{Ag}_{8}, \mathrm{Ag}_{10}$ or $\mathrm{Ag}_{16}$ ) whose fluorescence can be tuned by its surrounding nucleobases $9,10,14-19$. To create an NCB, a dark AgNC is first synthesized in a C-rich DNA host (termed the NC probe), and a Grich overhang (termed the activator) is brought into close proximity of the AgNC (via target-probe binding, Supplementary Fig. S1) to activate its fluorescence (Fig. 1a-b) ${ }^{4-6,14,17}$. Being a low-cost probe that can be easily prepared in a single-pot reaction at room temperature ${ }^{8}$, NCBs have been applied to the detection of nucleic acids ${ }^{14,17,20}$, proteins ${ }^{21}$, small molecules ${ }^{22}$, enzyme activities ${ }^{16}$ and cancer $^{2}$ cells ${ }^{23}$.

Whereas new applications of NCBs are emerging across a broad range of disciplines, it is unclear what sequence features of the activators ultimately control the enhancement ratio and activation color of an NCB. To answer this fundamental question and to unleash the power of NCB in biosensing, we design and study "polar opposite twin" NCBs (hereafter, denoted as POT-NCBs). Polar opposite twins are similar in appearance, but with very different personalities. In NCBs, polar opposite twins refer to a pair of NCBs that differ only by a single nucleotide in their activators, but have drastically distinct activation intensities or colors. Whereas POTs hold the key to understanding the NCB activation processes, there is no effective way to rapidly scan the vast activator sequence space and identify the most extreme POT-NCBs.

Here we repurpose the next-generation sequencing (NGS) chips for high-throughput screening of fluorescent nanomaterials. In a single experiment, more than $10^{4}$ activator mutations can be evaluated based on their capabilities in fluorescence activation of a common NC probe (C55 in Fig. 1a). Although the fluorescence properties of tens to hundreds of silver nanocluster species templated in short DNA strands can be studied in DNA microarrays ${ }^{24}$ and robotic plates ${ }^{15}$, less than 3,000 DNA hosts have been investigated as of today using these methods. While NGS chips are repurposed for studying protein-nucleic acid interactions ${ }^{25-29}$, they have never been used for study, selection, and optimization of fluorescent nanomaterials. By screening more than 40,000 activator sequences on three Illumina MiSeq chips, we not only discover new NCBs that are brighter than the known best (G15 and G12) but also identify the positions of nucleobases that are key to stabilizing bright AgNC chromophores (termed the critical zone). In the search for the most extreme POTs, the chip platform helps pinpoint the singlenucleotide substitution hotspots for generating yellow and red POT-NCBs, reaching 31-fold and 9-fold differences in the enhancement ratios, respectively (563 vs. 18 for a pair of yellow POTs and 285 vs. 32 for a pair of red POTs). Based on the findings of the critical zone for hosting bright chromophores (positions 7-12) and the hotspots for generating POTs (positions 4-6 for yellow POTs and positions 2-4 for red POTs), we propose a "zipper bag model" that explains how POT hotspots lead to the creation of distinct AgNC chromophores and contribute to the difference in chromophore chemical yields. In addition, with proper selection of the sequence features, we build machine learning models that can rationally design yellow and red NCBs. NCBs designed using these tools are 8.5 times and twice more likely to be bright yellow and red, respectively, as compared to the ones with random activator sequences. From our models, we create a new yellow NCB (with activator GTGTTGGGTGGTCGGGGG) that is twice as bright as the yellow standard G15 NCB, and a new red NCB (with activator ATCCCTCGGGGAGGGGGC) that is 1.3-fold brighter than the red standard G12 NCB. Our high-throughput screening and machine-learning-based design pipeline is not only 
accelerating the discovery of new NCBs for diverse applications, but also providing insights into the chemical yield and the emitter brightness controlled by the sequence features.

\section{Results}

\section{High-throughput selection of red and yellow NCB on NGS chips}

Our activator libraries were designed by systematically randomizing the canonical 18-nt-long activator G15,5,14,17 (GGGTGG GGTGGG GTGGGG, Fig. 2a and Supplementary Table S1). Together with the fiducial markers (PhiX), the library sequences were immobilized, bridge amplified and sequenced on each of the Illumina MiSeq chips. As the sequencing-needed barcodes and adapters (i.e., SP2/barcode/P7 adapter, Supplementary Table S1) could suppress or alter the NCB fluorescence (Supplementary Fig. S6-S8), they were removed using a restriction enzyme, leaving behind 20-nt-long activators (the library) that were only $2 \mathrm{nt}$ (CG dinucleotides) longer than the activators used in the traditional low-throughput test-tube selections ${ }^{4,5,14,17}$ (Fig. 1c and Supplementary Table S1). After enzymatic cleavage, the quality of the library was checked by staining the library with an Atto647N-labeled probe, before using the library for NCB selections (Supplementary Fig. S6).

We first aimed to discover new activators that light up the common C55 NC probe ${ }^{5}$

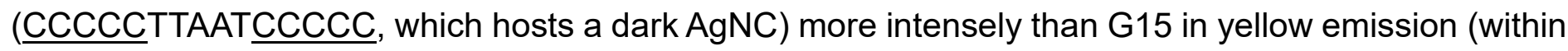
570-640 nm). We also searched for activators that give distinct emission colors (e.g., red emission within 663-738 nm). Once the complementary AT sequences on the C55 probe and the activators hybridized, AgNC emission developed ${ }^{5,6}$. In the traditional test-tube experiments, the NC probeactivator mixtures were heated to $90-95^{\circ} \mathrm{C}$ for a minute and gradually cooled down to room temperature to allow for hybridzation ${ }^{4,5}$. On the MiSeq chip, a constant temperature of $40{ }^{\circ} \mathrm{C}$ was maintained to enable hybridization (Supplementary Fig. S9; Methods). This relatively low hybridization temperature extended the life of the chip, allowing us to go through at least 20 rounds of activation experiments on a single chip (hybridized with C55 probes, washed and imaged, and then removed C55 probes using alkaline solution) without showing any degradation, thus providing highly reproducible selection results (Spearman's $\rho=0.93 \pm 0.005$ for the red NCBs and $0.88 \pm 0.024$ for the yellow NCBs, Supplementary Fig. S10-S11).

After injecting the common C55 NC probes into the MiSeq chip, the entire chip was scanned using a wide-field fluorescence microscope equipped with a metal halide illuminator, an sCMOS camera and an xyz translation stage. The activated NCBs were sequentially imaged using the filter cubes designed for conventional red emitters (e.g., Cy5, Ex/Em: 620/60, 700/75 nm) and yellow emitters (e.g., TRITC, Ex/Em: 535/50,605/70 nm). These two filter cubes were selected due to their popularity in fluorescence imaging. A custom bioinformatics and imaging processing pipeline ${ }^{28}$ was employed to identify activator sequence behind each activated NCB spot (Fig. 1c). After ranking the activators based on their median activation brightness (each activator had $457 \pm 308$ polonies on a MiSeq chip), we could clearly distinguish strong activators from weak ones in the two emission channels (Fig. 1c right).

Compared to other high-throughput screening methods that relied on fluorescence from single molecule for characaterization ${ }^{30}$, photobleaching was not a severe issue in our approach, as each 
activator polony contained tens to hundreds of activated NCBs. Besides, by employing an auto-scan algorithm and shutter control (Methods), excitation dose to each polony was precisely regulated, avoiding any uneven photobleaching and ensuring consistent imaging conditions. By acquiring a fluorescence image every $200 \mathrm{~ms}$, intensity time traces of polonies were obtained, which could be fitted with a single-exponential decay. After one second of strong illumination $\left(\sim 10 \mathrm{~W} / \mathrm{cm}^{2}\right)$, polony intensity decreased by $\sim 20 \%$ at most (Supplementary Fig. S13). Based on the chip screening results, twenty top-ranked and twenty bottom-ranked activators were selected for further investigation in test tubes by traditional fluorometry. Using G12 activator (ATCCGGGGTGGGGTGGGG) as the standard for red NCB comparison, the MiSeq chip screening results were $85 \%$ accurate in both true positive and true negative selections (brighter or darker than G12 in chip screening and confirmed in fluorometry, Fig. 1d, Supplementary Fig. S22-S23, and Supplementary Table S2). When comparing the chip screening results with the test-tube results, a Pearson correlation coefficient of 0.50 was obtained (Fig. 1d). We noticed the intensity differences found on MiSeq chips were substantially smaller than those found in test tubes (e.g., 1.20-fold red-emission difference was found between rAct1 and G12 NCBs on a MiSeq chip, but it became 2.94-fold in test tubes). The underestimation was attributed to the relatively higher fluorescence background on MiSeq chips and the variations in polony numbers among the library sequences (for instance, $\sim 240$ activators had less than 30 polonies). In a separate experiment, we selected ten activators there were found brighter than G15 (the standard for yellow NCB comparison) in MiSeq chips. All ten activators were still brighter than $\mathrm{G} 15$ in test tubes. In particular, we found yAct4 (TTGGTGGGTGGGGTGGGG) 2.03-fold brighter than G15 in activating C55 in the yellow channel (enhancement ratios were 1,125 vs. 553, Supplementary Fig. S24 and Supplementary Table S3) and rAct1 (TCCATTGGTGGGGTGGGG) 2.94-fold brighter than G12 in the red channel (enhancement ratios were 1,292 vs. 439, Fig. 1e, Supplementary Fig. S22 and Supplementary Table S2). We emphasize that the small-scale investigations carried out in test tubes, such as single-nucleotide substitutions from G15 at each nucleobase position (totally $3 \times 18=54$ variants), would not lead to any activators that are significantly brighter than G15 (Supplementary Fig. S18).

\section{Identification of critical nucleobases in stabilizing bright AgNC chromophores}

In our first library design (library_1, Supplementary Table S1), the 18-nt-long canonical G15 activator was divided into three 6-nt-long segments, and each segment was separately randomized to create $3 \times 4^{6}-2=12,286$ activator mutations (two were $\mathrm{G} 15$ duplicates in $3 \times 4^{6}$ combinations, Supplementary Table S1). The chip screening results on library_1 clearly indicated that segment_2 prefers to be conserved (GGTGGG) in order to maintain NCB brightness (Fig. 2). In contrast, randomizing segment_1 still produced many bright red NCBs, especially when segment_1 became Crich. The effect of segment_3 was diverse, suggesting an indirect activation role. The 6-segment interrogation further revealed that segment_22 (positions 10-12) is more important than segment_21 (positions 7-9) in creating bright red NCBs (Fig. 2c and Supplementary Fig. S14). Independent investigations using library_2 and library_3 ( 28,000 frame-shifted activators, Supplementary Table S1) also confirmed that the nucleobases in positions 10-12 are critical in C55 activation (Supplementary Fig. S14). These results indicated that bright AgNC chromophores are most likely 
"clamped" by the two strands at positions 7-12 (Fig. 2b), possibly forming silver-mediated pairs between the two strands ${ }^{31,32}$.

Drawing from the Fig. 2 results, one possible design rule for creating bright red NCBs on C55 could be having an activator with a C-rich segment_1, a GC-rich segment_21, a G-rich segment_22, and a TC-rich segment_3. Nevertheless, the activator CCCCCCGCGGGGTTTCCC (termed G5) actually had a low red enhancement ratio (39, as compared to 439 for G12; Supplementary Fig. S29 and Supplementary Table S8). This result clearly indicated that segments do not work alone cooperativities among the segments determine the activation color and intensity of an NCB. Whereas previous investigations showed that more guanines in the activator generally leads to brighter red emission ${ }^{4}$, our large-scale investigations revealed a different design rule - brighter red NCBs can be achieved with fewer numbers of guanines (e.g., 10G_5 in Supplementary Fig. S25 and Supplementary Table S4). As the results from our high-throughput screening could not be easily transformed into simple design rules, we trained machine learning algorithms on the big data and used them to rationally design bright NCBs (see Discussion).

\section{Discovery of polar opposite twins using NGS chips}

Taking advantage of the chip screening platform, we searched for POT-NCBs that have the most extreme color or intensity differences (Fig. 3a-c). Although NCBs were previously used for singlenucleotide polymorphism detection ${ }^{5,14}$, only tens of activators were tested, providing little information on the rules to design POTs. In contrast, library_1 alone contained more than 110,000 pairs of twin NCBs, where the top 2,000 pairs were readily candidates for POTs. Upon examining these 2,000 pairs of twin NCBs (Fig. 3d), it was clear that the nucleobases in positions 4-6 are critical for creating yellow POTs (e.g., bright (x-axis) $\rightarrow$ dark (y-axis) conversion by $\mathrm{G} / \mathrm{T} \rightarrow \mathrm{C} / \mathrm{A}$ substitution at position 5 and $\mathrm{G} \rightarrow \mathrm{C}$ substitution at positions 4 and 6), while the positions 2-4 are critical for creating red POTs (bright $\rightarrow$ dark conversion by $\mathrm{C} \rightarrow$ ATG substitution). Fifteen top POT pairs were further investigated in test tubes. The most extreme yellow and red POTs had 31-fold (yPOT5-yPOT6 with $\mathrm{G} \rightarrow \mathrm{C}$ substitution at position 5 ) and 9-fold (rPOT5-rPOT6 with $\mathrm{C} \rightarrow$ T substitution at position 4) differences in their enhancement ratios, respectively (Fig. 3e). For the ease of comparison, we termed the difference in the enhancement ratios the "POT difference ratio" (Supplementary Fig. S30-S31 and Supplementary Table S10-S11), where the pairs with the largest POT difference ratios are the most extreme POTs.

The POT difference ratio reflected the sample brightness difference at the ensemble level, which is equal to the product of "chromophore chemical yield ratio" and "single-emitter brightness ratio". Using fluorescence correlation spectroscopy (FCS) ${ }^{33,34}$, we found the chromophore chemical yield of rPOT5 NCB 5.54-fold higher than that of rPOT6 NCB (20\% vs. 3.6\%), and the single-emitter brightness of rPOT5 1.64-fold higher than that of rPOT6 (5.67 kHz vs. $3.45 \mathrm{kHz}$, Supplementary Fig. S16). The product of the $5.54 \times$ chromophore chemical yield ratio and the $1.64 \times$ single-emitter brightness ratio was indeed the 9x POT difference ratio measured by fluorometry. Similarly, the chromophore chemical yield of yPOT5 NCB was 16.33-fold higher than that of yPOT6 NCB (25.8\% vs. 1.6\%) and the singleemitter brightness of yPOT5 NCB was 2.17-fold higher than that of yPOT6 NCB (7.04 kHz vs. $3.24 \mathrm{kHz}$, Supplementary Fig. S17). The product of the $16.33 \times$ chromophore chemical yield ratio and the $2.17 \times$ 
single-emitter brightness ratio $(35 \times)$ was close to the $31 \times$ POT difference ratio measured at the ensemble level. Our investigation of POTs led to two important findings. First, the red AgNCs in rPOT5 and rPOT6 were actually different species as their excitation peak wavelengths (610 vs. $605 \mathrm{~nm}$, Supplementary Fig. S30a), absorption spectra (a clear peak at $610 \mathrm{~nm}$ in rPOT5 NCBs spectrum but no clear peak in rPOT6 NCBs spectrum, Supplementary Fig. S19d), and single-emitter brightness were all different. Second, the chemical yield of AgNC chromophores could be significantly altered by substituting single nucleobases at positions (2-6 in Fig. 3d) outside the critical zone (7-12 in Fig. 2). Based on these findings, we proposed a zipper bag model that explains the mechanism behind POT formation (Fig. 3a).

\section{Discussion}

\section{Investigation of the zipper bag model}

In our zipper bag model, the bag is the critical zone (positions 7-12) that holds the AgNC chromophore while the zipper is the POT hotspot that seals the bag. A subtle change in the zipper can alter the sealing condition of the zipper bag, which perturbs the short-range ligand environment around the AgNC inside the bag and possibly changes its binding footprint with the bag (Fig. 3a). We have previously shown that by slightly shifting the position of the activator with respect to the NC probe, a new ligand environment can be created around the AgNC that alters its emission spectrum ${ }^{14,17}$, and we believe such a nucleobase-AgNC interaction is within a short range $(\leq 1 \mathrm{~nm})^{14}$. When studying AgNC structures using $193 \mathrm{~nm}$ activated-electron photodetachment mass spectrometry (a-EPD MS), we have previously found two $\mathrm{Ag}_{10}$ clusters can be completely distinct chromophores due to very different binding footprints in their DNA hosts ${ }^{35}$. The earlier structural studies by extended X-ray absorption fine structure (EXAFS) spectra complemented the a-EPD MS footprint results ${ }^{11,36}$. Here by using FCS, we further demonstrated that a change in zipper may result in not only a distinct AgNC chromophore in the bag but also a different chemical yield of the chromophore, thus providing a basis for POT formation.

In our model, the zipper may not necessarily be formed by the Watson-Crick (WC) basepairs - it can also be formed by the silver-mediated pairs (e.g., the $\mathrm{C}-\mathrm{Ag}^{+}-\mathrm{C}$ pair) ${ }^{31,32}$. Upon close examination, we believe the yellow POT zippers at positions 4-6 are caused by a WC pair GC or a wobble pair GT (where $\mathrm{G}$ is on the activator and $\mathrm{C}$ or $\mathrm{T}$ is on the NC probe), as disrupting the GC or GT pair at these positions often creates dim yellow NCB samples (Supplementary Fig. S31b). As aforementioned, these dim yellow samples attribute to less emissive AgNC chromophores and lower chemical yield of the chromophores. In contrast, the red POT zippers at positions 2-4 may be formed by a silver-mediated pair $\mathrm{C}-\mathrm{Ag}^{+}-\mathrm{C}$. Disrupting the $\mathrm{C}-\mathrm{Ag}^{+}-\mathrm{C}$ pair at these positions darkens red NCB samples (Supplementary Fig. S30b). One recent report showed evidence that silver-mediated heteroduplexes (e.g., $\left.\mathrm{C}_{11}-\mathrm{Ag}^{+} \mathrm{N}-\mathrm{T}_{11}\right)$ could be less stable than their homoduplex counterparts (e.g., $\left.\mathrm{C}_{11}-\mathrm{Ag}^{+} \mathrm{N}-\mathrm{C}_{11}\right)^{31}, w \mathrm{while}$ another report showed a $\mathrm{Ag}^{+}$-mediated interaction at a place away from the $\mathrm{AgNC}$ core ${ }^{37}$, both supporting the hypothesis behind our model.

Why are the zipper locations different for the yPOT5-yPOT6 pair (at position 5) and the rPOT5rPOT6 pair (at position 4)? One possibility is red AgNC chromophores have higher silver stoichiometries and larger footprints in the bag, pushing the zipper locations further away from the bag (positions 7-12). 
Using electrospray ionization mass spectrometry (ESI-MS), Gwinn's group has previously shown a general trend for yellow chromophores having a smaller core (Ag10-Ag11) while red chromophores having a larger core $\left(\mathrm{Ag}_{14}-\mathrm{Ag}_{16}\right)^{10}$. According to their rod-shaped model $^{9}$, red chromophores are expected to have larger footprints in their DNA hosts. However, in Gwinn's experiments, their AgNC chromophores most likely stabilized inside dimers of 10-mers, which create ligand environments that are different from our activator/NC probe systems. To investigate the silver stoichiometries of the two major chromophores in our POT experiments, we purified yPOT5, yPOT6, rPOT5 and rPOT6 NCB samples using 20\% native polyacrylamide gel electrophoresis (native PAGE) (Supplementary Fig. S20; Methods) and analyzed the purified samples by ESI-MS (Supplementary Fig. S21). Upon close examination of the gel shift results, yPOT5 NCB clearly had a higher mobility as compared to rPOT5 NCB, possibly due to a more condensed form of the chromophore-DNA complex. Similar results were also observed for the yellow standard G15 NCB, which ran faster than the red standard G12 NCB in gel. We also noticed that the brighter NCBs (yPOT5 and rPOT5) had slightly higher mobility than their dim counterparts (yPOT6 and rPOT6), which could infer that the activators of the dim chromophores are "wrapped" loosely around the C55 NC probe and hence slightly larger complexes were formed.

Since our NCB constructs were much larger (45-nt long for the NC probe strand and 48-nt long for the activator strand) than the constructs used in previous studies involving ESI-MS analysis ${ }^{10,38,39}(10-$ to 26-nt-long), extensive cationic metal adduction during the ESI process prevented us from deciphering the exact silver stoichiometries in purified NCB samples. To circumvent this issue, an ionpairing reagent, octylamine, was added to the ESI samples to suppress salt adduct formation ${ }^{40-43}$. Although the number of adducts was reduced, the addition of octylamine destabilized the duplex NCB structures. ESI-MS of purified yPOT5 NCB and rPOT5 NCB samples containing octylamine showed clear peaks of single-stranded species (i.e., C55 NC probe and activator) with various silver stoichiometries (Supplementary Fig. S21). Whereas it was still difficult to conclude the exact silver stoichiometries in the original duplexes, introducing octylamine to the MS spray solution led to wellresolved single-stranded species with highly reproducible numbers of silver atoms on them. The C55 NC probes from yPOT5 NCB and rPOT5 NCB carried 0-4 and 0-3 silver atoms, while the activators yPOT5 and rPOT5 carried 0-7 and 0-6 silver atoms, respectively. These results indicated that the original silver stoichiometry for the intact yPOT5 NCB may be larger than that of the intact rPOT5 NCB. Although our results are contradictory to previous investigations that suggest red AgNC chromophores have a larger core ${ }^{10}$, we predict that the red chromophores featured in this study have a larger footprint in the activator/C55 bag, owing to different AgNC shapes and DNA conformations ${ }^{9,12,13,35,44}$. In future studies, we aim to pinpoint the binding sites of AgNC chromophores within the activator/NC probe duplexes by employing activated-electron photodetachment mass spectrometry (a-EPD MS), a structural characterization technique and tandem $\mathrm{MS}^{\mathrm{n}}$ method that was previously shown to reveal the binding sites of AgNCs in shorter single-stranded DNA hosts (up to 28-nt long) ${ }^{35}$. Alternatively, X-ray crystallography of DNA-templated AgNCs can reveal not only the binding sites but also the binding geometries of surrounding bases to $\operatorname{AgNCs}^{12,13,37,44}$, provided that NCBs can be crystallized. 
Since the results from our high-throughput screening (Fig. 2) cannot be easily transformed into simple design rules, we take advantage of machine learning algorithms to classify NCBs and uncover sequence features that give bright NCBs. Machine learning approaches have previously helped identify sequence features in DNA hosts that preferentially stabilize bright AgNCs, establishing the first statistical model for rational design of AgNCs with desired colors ${ }^{15,45}$. However, such a model was built upon the emission properties of $\sim 2,000$ AgNCs sandwiched between two identical strands (8-mers to 16-mers) ${ }^{46}$. Our AgNCs were different, as each of them was stabilized within an 18-nt long activator and a 15-nt long C55. Although both robotic-well-plate studies ${ }^{46}$ (2,000 short strands) and NGS screening $(40,000 \mathrm{NCBs}$ in this report) covered only a small fraction of the overall ligand composition space of 10-mers and 18-mers, respectively, statistical models could be built based on these small fractions of data. This was due to the fact that a majority of the ligand composition space was only occupied by dark AgNCs. Previous selection and our screening here focused heavily on the sequences that had a high chance to produce bright AgNCs, thus giving a higher "effective" fraction of search space.

To connect activator sequences to NCB brightness, we adopted machine learning algorithms to recognize sequence features (which consist of motifs and motif locations) in the bright activators. Following the approaches proposed by Copp and Gwinn ${ }^{15,45,46}$, we labeled the top $30 \%$ NCBs $(3,600)$ as "bright" class and the bottom $30 \%$ as "dark" class. 339 and 567 features from the bright and dark classes in the yellow channel (denoted as bright yellow and dark yellow features) and 402 and 1,164 features from the bright and dark classes in the red channel (denoted as bright red and dark red features) were separately identified by $\mathrm{MERCI}^{47}$. To decrease the chance of overfitting, we further narrowed down to a set of the most discriminative features with 61 bright yellow, 121 dark yellow, 103 bright red and 112 dark red features using Weka ${ }^{48}$ (Supplementary Table S13-S14; Methods). A feature vector was employed to describe the location of each activator in the high-dimensional space for classification model development (Fig. 4a).

A number of models were established for classifying the chip screening results, based on algorithms such as logistic regression (LR), linear discriminant analysis (LDA), decision tree (DT), AdaBoost (ADA), and support vector machines (SVM) (Supplementary Table S12). To evaluate the model performance, we defined the accuracy of the model $(A c c)$ as $\left(T_{B}+T_{D}\right) /\left(T_{B}+F_{B}+T_{D}+F_{D}\right)$, where $T_{B}$ is the number of true predictions that the model makes for bright activators, $T_{D}$ and $F_{D}$ are the numbers of true and false dark predictions, and $F_{B}$ is the number of false bright predictions. In other words, Acc represented the fraction of test sequences that the model correctly identifies as "bright" or "dark" activators. We found the model built on LR has the best performance, achieving an average accuracy of 0.89 and 0.87 in the yellow and red emission classification, respectively. In our model development process, the categorized dataset was divided into a training set ( $80 \%$ of the selected sequences) and a test set (20\% of the selected sequences). The selection process iterated 5 times while rotating the training set and the test set, resulting in a 5-fold cross-validation (CV) that guarantees the model consistency (Supplementary Fig. S34). As expected, the propensity of an activator to be "bright" is not only determined by having "bright" motifs within the activator but also by positioning these motifs at proper locations. 
Separately, based on the most discriminative features identified by Weka ${ }^{48}, 1,000$ bright yellow and 1,000 bright red activator candidates were rationally designed in silico (Supplementary Fig. S35; Methods). In the high-dimensional space, we employed the minimal "edit distance" 46,49 to identify the "closest" activator sequence in the library dataset for each of the rationally designed candidates. When the closest library sequence was not among the top 200 bright activators screened, the candidate was discarded. Besides, when the candidate sequence had less than 3 or more than 5 single-base mutations, they were also discarded. After going through these candidate refining steps, we were down to 100 bright yellow and 54 bright red candidates. Among these candidates, the LR model classified 85 and 41 of them as bright yellow and red activators, respectively. From these most promising candidates, 20 were randomly selected, synthesized, hybridized with the C55 probes in test tubes, and measured by a fluorometers.

Compared to the random activator sequences (Supplementary Fig. $\mathbf{S 2 8}$ and Supplementary Table S8), our design and classification pipeline generated new activators that were 8.5 times (85\% vs. $10 \%$ ) and twice ( $85 \%$ vs. $40 \%$ ) more likely to be bright yellow and red activators, respectively (Supplementary Fig. S26-S27 and Supplementary Table S6-S7). Besides, the average enhancement ratio of rationally designed yellow and red activators were 22 times and twice higher than that of random sequences, respectively (Supplementary Table S8). Moreover, while all random sequences gave more or less red emission, our pipeline successfully produced NCBs with yellow emission (Fig. 4b). In particular, among all bright candidates tested, we identified a new yellow activator (yPred1: GTGTTGGGTGGTCGGGGG, with only 12 guanines) and a new red activator (rPred3: ATCCCTCGGGGAGGGGGC, with only 9 guanines) that were 2.13-fold and 1.30-fold brighter than the gold standards G15 and G12 in activating the C55 NC probe, respectively (Fig. 4c, Supplementary Table S6-S7).

\section{Summary}

We have performed high-throughput screening on more than 40,000 activators using repurposed NGS chips. Not only did we discover new NCBs (yAct4 and rAct1) that are 2-3 times brighter than the known best (G15 and G12, Fig. 1), but we also identified a critical zone in the activator (positions 7-12) that stabilizes bright AgNC chromophores (Fig. 2). In the search for the most extreme POT-NCBs, the chip platform helped identify a red pair (rPOT5-rPOT6) and a yellow pair (yPOT5-yPOT6) with POT difference ratios as high as 9 and 31, respectively (Fig. 3). By probing the NCBs at the near singlemolecule level, we confirmed the observed brightness difference at the ensemble level is attributed to the differences in the single-emitter brightness and the chromophore chemical yield (Supplementary Fig. S16-S17). Based on the findings of the critical zone (positions 7-12) and the POT hotspots (positions 2-4 for the red POTs and positions 4-6 for the yellow POTs), we proposed a zipper bag model that explains how POT hotspots lead to the creation of distinct AgNC chromophores and contribute to the difference in chromophore chemical yields (Fig. 3). As the results from high-throughput screening could not be easily converted into simple rules for designing bright NCBs, we employed machine learning algorithms to classify the screening results and used the trained model to rationally design multicolor NCBs. Forty new NCBs were generated, clearly showing two designated color bands (Fig. 
4). We also found brighter NCBs could be achieved with fewer numbers of guanine bases in the activators. Our chip screening platform can facilitate the development of new chemical sensors based on DNA-templated $\mathrm{AgNCs}^{50}$ or be used to study other metal nanoclusters templated in DNA ${ }^{51-53}$. To our knowledge, this article is the first report that NGS chips are repurposed for high-throughput screening of fluorescent nanomaterials. Our high-throughput screening and machine-learning-based design pipeline is not only accelerating the discovery of new NCBs for diverse applications, but also providing insights into the chemical yield and the emitter brightness controlled by the sequence features. We anticipate new NCB-based sensors and new fluorescence barcodes will soon be developed based on the design strategy that we layout in this article.

\section{Methods}

\section{NC probe preparation}

Sodium phosphate dibasic anhydrous ( $\mathrm{Na}_{2} \mathrm{HPO}_{4}$; F.W. 141.96), sodium phosphate monobasic monohydrate $\mathrm{Na}_{2} \mathrm{HPO}_{4} \cdot \mathrm{H}_{2} \mathrm{O}$; F.W. 137.99) and sodium borohydride $\left(\mathrm{NaBH}_{4}\right)$ were purchased from Fisher Scientific, whereas silver nitrate $\left(\mathrm{AgNO}_{3}\right)$ was acquired from Sigma-Aldrich. All oligonucleotides were purchased from Integrated DNA Technologies (IDT) and were purified by desalting. Deionized (DI) water $(18 \mathrm{M} \Omega \cdot \mathrm{cm})$ was used for all solution preparations.

In a typical preparation, a $15 \mu \mathrm{M}$ (final concentration) NC probe solution was prepared by adding $12.5 \mu \mathrm{l}$ of $1.2 \mathrm{mM} \mathrm{NC}$ probe (C55, Supplementary Table S1) to $940 \mu \mathrm{l}$ of $20 \mathrm{mM}$ sodium phosphate buffer (pH 6.6). The solution was vortexed for $2 \mathrm{~s}$, and $45 \mu \mathrm{l}$ of $4 \mathrm{mM}$ silver nitrate solution was added to it. Again, the mixture was vortexed for $2 \mathrm{~s}$. The solution was allowed to sit in the dark for $10 \mathrm{~min}$ at room temperature. For silver cluster formation, $7 \mu \mathrm{l}$ of freshly prepared $13.2 \mathrm{mM} \mathrm{NaBH}_{4}$ solution was added to the reaction, resulting in a pale-yellow mixture, which was then stored in the dark overnight. The resulting NC probe solution had the [NC probe]: $\left[\mathrm{Ag}^{+}\right]$: $\left[\mathrm{NaBH}_{4}\right]$ molar ratio of 1:12:6.

Before MiSeq chip experiment, a $0.5 \mathrm{ml}$ centrifugal filter (cat. no. UFC503024, MilliporeSigma) was employed to remove excess silver ions. Purification protocol followed the manufacturer guidelines. The filtered solution was then diluted to $500 \mathrm{nM}$ (DNA concentration, which was verified using the NanoDrop 2000 UV-Vis spectrophotometer, Thermo Scientific) before being injected into MiSeq chip.

\section{NCB preparation and in-solution validation}

To activate NCB, $1.5 \mu \mathrm{l}$ of $1.2 \mathrm{mM}$ activator solution was added to a $120 \mu \mathrm{l}$ aliquot of the previously prepared $15 \mu \mathrm{M}$ C55 probe solution. The mixture was vortexed, centrifuged, and immersed in a hot water bath $\left(90-95^{\circ} \mathrm{C}\right)$ for $1 \mathrm{~min}$, followed by gradually cooling down to room temperature for $1 \mathrm{hr}$. The activated NCB had the [NC probe]: [activator] molar ratio of 1:1. The fluorescence measurements started exactly at $1 \mathrm{hr}$ after the addition of activator.

We quantified NCB fluorescence using a fluorometer (FluoroMax-4, Horiba) and a $100 \mu \mathrm{l}$ quartz cuvette (16.100F-Q-10/Z15, Starna Cells). Both the excitation and emission wavelength scan ranges were set to be from $400 \mathrm{~nm}$ to $800 \mathrm{~nm}$ using $5 \mathrm{~nm}$ slit size, $5 \mathrm{~nm}$ increment step, $0.1 \mathrm{~s}$ integration time. Two control samples, a 20 mM sodium phosphate pH 6.6 buffer only sample and an NC probe only sample (with AgNCs but no activators) were also measured. The acquired spectra were saved as csv 
files and processed using a Python script.

\section{NCB fluorescence enhancement ratio calculation}

We followed a similar definition described in ref. 5 to calculate the ensemble enhancement ratio of NCB after activation. However, in ref. 5, 1D spectra based on $580 \mathrm{~nm}$ excitation were acquired and area integrated intensities were calculated over 595 to $740 \mathrm{~nm}$ emission range. Here we collected 2D spectra of samples and calculated volumetric integrated intensities over the red (Ex/Em: 620/60, 700/75 nm) and the yellow (Ex/Em: 535/50, 605/70 nm) excitation/emission "windows" (Supplementary Fig. S2). From there we calculated the enhancement ratio:

$$
\text { Enhancement ratio }=\frac{\left(I_{N C B}-I_{\text {background }}\right)-\left(I_{N C \text { probe }}-I_{\text {background }}\right)}{I_{N C \text { probe }}-I_{\text {background }}}=\frac{I_{N C B}-I_{N C \text { probe }}}{I_{N C \text { probe }} I_{\text {background }}}
$$

, where INCB stands for the volumetric integrated intensity of NCB in red or yellow window, INC probe represents the volumetric integrated intensity of dark AgNC on the C55 probe, and lbackground is the volumetric integrated intensity of the sodium phosphate buffer. The improvement ratio is simply the ratio of the enhancement ratio of an activator to that of the standard activator (G12 or G15). Similarly, the POT difference ratio is simply the ratio of the enhancement ratios of the twins:

POT difference ratio $=$ (enhancement ratio of bright twin NCB) / (enhancement ratio of dark twin NCB)

\section{NCB fluorescence visualization}

Color photos of inactivated (NC probe only) and activated (the duplex) NCBs were acquired using a digital camera (PowerShot SX 500 IS, Canon) on a Syngene gel imager (with $365 \mathrm{~nm}$ excitation) (Fig. 1b). NCB fluorescence were also visualized using a gel imaging scanner (Typhoon 9500, GE Healthcare Life Sciences). For the Typhoon 9500 experiments, $240 \mu$ of NCB sample was placed in a single well on the 96 multi-well plate. The fluorescence was acquired using the built-in Cy3 channel (EX: $532 \mathrm{~nm}$, EM: $575 \mathrm{~nm}$ long pass) or the Cy5 channel (EX: $635 \mathrm{~nm}$, EM: $665 \mathrm{~nm}$ long pass) while the PMT gain was set to 400 and the pixel size was $10 \mu \mathrm{m}$. The imaging results were saved as tiff files and changed to 16-bit false colors (yellow for Cy3-channel imaging and red for Cy5-channel imaging) using ImageJ.

\section{Activator library design}

All activator strands contained a universal, 30-nt-long TA-rich hybridization segment followed by an 18nt-long variable region (the activator) and an 8-nt-long restriction site (Supplementary Table S1). Additional adapters at 5'-end (P5 and SP1) and 3'-end (P7 and SP2) were designed by Illumina for sequencing purpose. To identify our library sequences, a 6-nt-long barcode was also needed and that was added to the 3'-end. The barcode for the canonical activator G15 was different from that of any other activators in order to monitor the sequencing yield. Three different libraries were established based on shifted frames, giving totally 40,068 unique activator sequences (Supplementary Table S1).

\section{NGS library preparation}


A standard PCR process was performed using Q5 high-fidelity DNA polymerase from NEB (cat. no. M0491S). All PCR primers were purchased from IDT (Supplementary Table S1). The PCR procedure and the thermal cycler (Eppendorf, Mastercycler ${ }^{\circledR}$ nexus) settings followed the protocol provided by NEB. The library sequences and the canonical activator were amplified separately, reaching a final concentration greater than $5 \mathrm{ng} / \mu \mathrm{l}$ for each tube. After PCR amplification, the concentration of DNA library was verified using a NanoDrop 2000 UV-Vis spectrophotometer (Thermo Scientific). Together with the fiducial markers (PhiX), the library sequences were immobilized and bridge amplified on an Illumina MiSeq chip, followed by sequencing using a $2 \times 300$ paired end reagent kit (v3, Illumina). For the 3-segment interrogation (Fig. 2), we targeted to have 1.2 million reads for the mutations in each segment. 10,000 additional reads were expected for the canonical activator $\mathrm{G} 15$. The fiducial markers, PhiX, were counted for $15 \%$ 20\% of the overall coverage. The actual numbers of reads varied from batch to batch, but were within 5-13 million reads.

\section{MiSeq chip preparation}

After sequencing, MiSeq chips were kept at $4^{\circ} \mathrm{C}$ in storage buffer (1X TBE buffer, cat. no. AM9865, Invitrogen). Before hybridizing with NC probes, all DNA strands covalently affixed to the MiSeq chip surface were denatured with $20 \mu \mathrm{l} 0.1 \mathrm{~N} \mathrm{NaOH}$ solution for 5 min and then rinsed with $20 \mu \mathrm{l}$ 1X TBE buffer 3 times to remove excess $\mathrm{NaOH}$. This rinsing step removed untethered DNA strands containing residual fluorescent dyes from sequencing. Before the NCB screening experiment, chip was rinsed with working buffer $(150 \mu \mathrm{l}, 200 \mathrm{mM}$ sodium phosphate buffer $\mathrm{pH} 6.6)$ three times. To cleave the unwanted sequence beyond the activator sequence, a 32-nt-long strand complementary to the restriction site (RE strand in Supplementary Table S1) was introduced to the chip and the chip was annealed at $40{ }^{\circ} \mathrm{C}$ for 40 min. After annealing, 1X TBE buffer was used to rinse the chip, followed by MauBI (cat. no. ER2081, Thermo Fisher Scientific) restriction enzyme digestion. The reaction buffer was prepared following the manufacturer's protocol and $20 \mu$ lenzyme solution was kept in the MiSeq chip at $40{ }^{\circ} \mathrm{C}$ for $40 \mathrm{~min}$. After digestion, the chip was washed with $20 \mu \mathrm{l} 0.1 \mathrm{~N} \mathrm{NaOH}$ solution for 5 min and rinsed with $20 \mu \mathrm{l} 1 \mathrm{X}$ TBE buffer for three times. The PhiX sequences were labeled using an Atto488-tagged probe (500 nM and $20 \mu$, Supplementary Table S1) for fiducial marker imaging. To optimize the annealing conditions, we evaluated the intensities of NCBs that had gone through different temperature treatments $\left(40{ }^{\circ} \mathrm{C}\right.$ for 40 min, room temperature for $40 \mathrm{~min}$, and $90{ }^{\circ} \mathrm{C}$ for $10 \mathrm{~min}$, Supplementary Fig. S9). Holding the chip at $40{ }^{\circ} \mathrm{C}$ for 40 min not only gave an excellent annealing result but also extended the chip life to up to 20 runs of NCB activation experiments. After testing different concentrations of C55 probes for the NCB screening experiment (Supplementary Fig. S12), we chose to use $20 \mu \mathrm{l}$ of $500 \mathrm{nM}$ C55 probe solution for all our chip experiments. The chip was imaged at room temperature with microscope settings stated below. After each experiment, the chip was washed with $20 \mu \mathrm{l} 0.1 \mathrm{~N} \mathrm{NaOH}$ solution for 5 minutes, followed by rinsing with $20 \mu \mathrm{l}$ X TBE buffer for three times and storing at $4{ }^{\circ} \mathrm{C}$.

\section{Fluorescence microscopy and image acquisition}

An open-source software, Micro-Manager ${ }^{54}$, was used to control an sCMOS camera (ORCA-Flash 4.0, Hamamatsu), an xyz translation stage (ProScan III, Prior Scientific), and an auto-shutter (Lambda SC, 
Shutter Instrument) on an Olympus IX71 fluorescence microscope for all our MiSeq screening experiments. A metal-halite illuminator (Lumen 200, Prior) and a $60 \times$ water-immersion objective (UPLSAPO60XW, Olympus) were used in the IX71 system. We developed a MATLAB script to generate the position list of each field of view for automatic acquisition. On each MiSeq chip, we acquired 60 images (FOV: $220 \times 220 \mu^{2}$ ) per row for 3 rows on both floor and ceiling (totally 360 images), covering a total surface area of $5.81 \mathrm{~mm}^{2}$. It is worthwhile to note that polonies in some regions of the chip were not registered in the Illumina sequencing files. To bypass most of these unregistered regions, we shifted the imaging starting position by $380 \mu \mathrm{m}$ in the vertical direction and 1,611 $\mu \mathrm{m}$ in the horizontal direction with respect to the reference point at the bottom left corner (Supplementary Fig. S3c). We first recorded fiducial marker images (Atto488, FOV: $220 \times 220 \mu \mathrm{m}^{2}, 1$ second exposure time, green channel, Ex/Em: 480/40, 535/50 nm, cat. no. 51006, Chroma), and then recorded NCB images in both red (Ex/Em: 620/60 nm, 700/75 nm, cat. no. 49006, Chroma) and yellow channels (Ex/Em: 535/50 nm, $605 / 70 \mathrm{~nm}$, cat. no. 49004 , Chroma) under the same imaging settings.

\section{Flat-field correction}

We implemented flat-field correction to eliminate the variation of fluorescence background across the field of view (FOV, Supplementary Fig. S3a-b). A Gaussian-blur filter was applied to generate the flatfield reference image for each FOV. We found that a Gaussian-blur filter with sigma equal to 50 best fit our purpose. The corrected imaged used for the following analysis were computed as follows:

$$
I_{\text {corrected }}=I_{\text {measure }} I_{\text {dark }} /\left(I_{\text {flat-field image }} I_{\text {dark }}\right)
$$

, where $I_{\text {measure }}$ is the recorded fluorescence images, $I_{\text {flat-field image }}$ is the flat-field reference image generated by the Gaussian-blur filter, and $I_{\text {dark }}$ is the dark image recorded using 1 second exposure time while illuminator is turned off.

\section{Fluorescence correlation spectroscopy (FCS) measurements}

FCS measurements were carried out using a confocal system (Alba v5, ISS) built around a Nikon microscope body (Eclipse TE2000-U, Nikon). A super-continuum laser (SuperK EVO EU-4, NTK Photonics) and a 60× water-immersion objective (CFI Plan Apochromat VC, Nikon) were used in the FCS experiments. To validate the NCB chemical yield measurement results, an Alexa647N-labeled ssDNA probe was used to generate the concentration calibration curve shown in Supplementary Fig. S16-S17. All FCS measurements were carried out using $200 \mu \mathrm{l}$ samples in 8 -well chamber slides (Nunc Lab-Tek, Thermo Fisher Scientific). The laser beam was focused $25 \mu \mathrm{m}$ into the sample for all FCS measurements in this work.

\section{FCS analysis}

Autocorrelation curves were fitted using the software package provided by ISS, giving estimates on the average number of emitters in the detection volume $(N)$ and the average translational diffusion time constant $(\tau)$. Single-emitter brightness (SEB) was computed based on:

$$
\mathrm{SEB}=\text { Average photon count rate }(\mathrm{kHz}) / \mathrm{N}
$$

The "activated" NC probe concentration was derived from the calibration curve established by the 
Alexa647N probe (Supplementary Fig. S16-S17). NCB chemical yield was then computed as:

NCB chemical yield $=($ Activated NC probe concentration in $\mathrm{nM}) \times($ dilution constant $) / 15,000$ , where 15,000 nM represents the DNA concentration in the original reaction (i.e., $15 \mu \mathrm{M}$ ).

\section{Absorption measurements}

Absorption spectra of NCBs were measured using Cary 5000 UV-Vis-NIR spectrometer from Agilent. $500 \mu \mathrm{l}$ of $15 \mu \mathrm{M}$ NCB solution was prepared following aforementioned protocol and was injected into a $700 \mu \mathrm{l}$ Micro Fluorescence Cuvette from Thorlabs. The dual-beam mode was used with baseline/zero correction. All absorption spectra were measured from $300 \mathrm{~nm}$ to $800 \mathrm{~nm}$ with slit size of $2 \mathrm{~nm}$ (Supplementary Fig. S19). The acquired data were processed and analyzed using Python scripts.

\section{Image alignment algorithm}

A custom bioinformatics and imaging processing pipeline named CHAMP (ㅡhip-ㅡybridized Associated Mapping Platform) was developed by Finkelstein's group and the detailed algorithm description can be found in ref. 28. CHAMP helped decipher the activator sequence behind each activated NCB spot (termed the NCB-CHAMP selection method, Fig. 1c and Supplementary Fig. S4-S5). In brief, mapping the alignment markers was done at four stages. First, a rough alignment was carried out using Fourierbased cross correlation, followed by a precision alignment using least-squares constellation mapping between FASTQ and de novo extracted NCB spots. We built up the consensus sequences and their corresponding information (e.g., lane number, tile number, and $x-y$ coordinates) at all reported positions in the FASTQ file using the map command. Second, the init command was executed to record the metadata of imaging settings (e.g., rotation and scaling). Third, the $\boldsymbol{h} 5$ command was applied to generate a single hdf5 file containing all $512 \times 512$ PhiX fiducial marker images. Fourth, the align command transformed the processed sequence information into pseudo-images and performed precise alignment. The output files were saved individually by image positions. The content included $x, y$ coordinates of each sequence and the corresponding sequence ID. To analyze our NCB images, we developed an additional function named $\boldsymbol{n c b}$, which corrected the uneven illumination using flat-field correction. A bootstrap method was then performed to derive the median intensity of each activator in order to rank the NCB brightness (Supplementary Fig. S36).

\section{Feature extraction/selection and machine learning model establishment/validation}

The feature extraction was performed using $\mathrm{MERCl}^{47}$. For all extraction processes, both positive and negative thresholds were set to $5 \%$, the maximal motif length was set to 6 bases, and the maximal number of wildcard nucleotide (A, T, G, C, or nothing) was set to 1 base. For example, in 5-fold crossvalidation, the threshold was set to be 144 ( $5 \%$ of 2,880 sequences for each class). Separately, to extract "bright" features, the entire bright and dark classes were used (3,600 sequences for each class) and 180 was set as the threshold. The dark feature extraction was performed by simply swapping the bright and dark classes with the same parameter settings. The extracted motifs were then processed with Python scripts to include the position information. 339 bright yellow, 567 dark yellow features, 402 bright red and 1,164 dark red features were separately identified. 
To decrease the chance of overfitting, we further narrowed down to a set of the most discriminative features with 61 bright yellow, 121 dark yellow, 103 bright red and 112 dark red features using Weka ${ }^{48}$ - a process we termed feature selection. The attribute evaluator was set to "CfsSubsetEval" 55 and the search method was set to "GreedyStepwise"48. CFS scored a feature subset based on high correlation of features with predictive classes and low inter-correlation of features ${ }^{56}$. The "greedy" algorithm started with an empty set and iteratively added the feature that maximizes the gain in the CFS score. Feature selection process stopped when any additional feature decreased the CFS score. All other parameters were set to default values (Supplementary Fig. S34).

After feature extraction and selection, classification models were established based on various $\mathrm{ML}$ algorithms including logistic regression (LR), linear discriminant analysis (LDA), decision tree (DT), AdaBoost (ADA), and support vector machines (SVM), using the scikit-learn package in Python. 5-fold cross-validation was performed to evaluate the model performance. The best model (i.e., LR model) was employed to rationally design bright and multicolor NCBs (Supplementary Fig. S34).

\section{In-silico design of bright NCBs}

To rationally design red and yellow NCBs, we again divided the 18-nt-long activator into 3 segments. Based on the most discriminative features identified by Weka, we sampled the distribution of these features in each segment and generated a list of common motifs with their corresponding positions. Please note that the features selected from each segment could slightly go beyond the range of that segment. To construct a red NCB candidate, we assigned 3 features to the blank 18-nt template, starting with feature_1 insertion into segment_1. As feature_1 could go beyond segment_1, feature_2 might have an overlap with feature_1 when being inserted into segment_2. In that situation, the design algorithm would replace feature_2 with another feature to ensure no overlap. However, if any two features shared identical bases at their overlapping site, they were considered as "compatible" and could be inserted into the same template. For example, as shown in Supplementary Fig. S35, feature C_CTG (positions 1-5) and feature GGG_GC (positions 5-10) shared a guanine base at the overlapping site (position 5). Consequently, they were compatible and were used in constructing a bright NCB candidate. The same procedure was repeated until a compatible feature for segment_3 was found. Once all three features were inserted into the template, the remaining blank positions were filled up based on the composition popularity (at the same positions) from the bright class sequences. The edit distance 46,49 of the new candidate was then assessed. We only selected new candidates with edit distance between 3 to 5 from the top 200 bright activators screened on chip for test-tube investigation (Supplementary Table S6 and S7).

\section{NCB mobility evaluation in native PAGE gels}

$30 \mu \mathrm{M}$ of dark C55 probes were prepared following the previously stated protocol. The same molar ratio of [NC probe]: $\left[\mathrm{Ag}^{+}\right]:\left[\mathrm{NaBH}_{4}\right]=1: 12: 6$ was used while doubling the amount of each chemical. $30 \mu \mathrm{M}$ NCB sample was prepared by adding $3 \mu \mathrm{l}$ of $1.2 \mathrm{mM}$ activator to the $120 \mu \mathrm{l}, 30 \mu \mathrm{M}$ C55 probe solution, followed by the aforementioned hybridization and buffer exchange protocol.

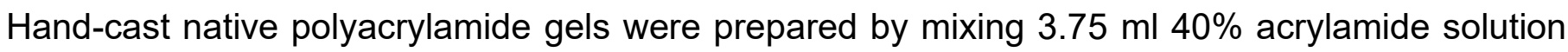


(cat. no. HC2040, Invitrogen), $0.75 \mathrm{ml}$ TBE 1X buffer (Invitrogen), $3 \mathrm{ml}$ DI water, $75 \mu \mathrm{ll} 10 \% \mathrm{w} / \mathrm{w}$ ammonium persulfate solution (cat. no. HC2005, Invitrogen), and 7.5 $\mu$ I TEMED (cat. no. 45-000-226, Fisher Scientific), targeting gel percentage at $20 \%$. Gel solution was then poured onto the $1.0 \mathrm{~mm}$ empty gel cassette (cat. no. NC2010, Life Technologies). The cured gel was pre-run at 60V, $5 \mathrm{~mA}$ for $30 \mathrm{~min}$ in $1 \mathrm{X}$ TBE buffer before loading any samples. The DNA ladder was prepared by mixing $4 \mu \mathrm{l}$ of $100 \mu \mathrm{M}$ 20/100 ladder (cat. no. 51-05-15-02, Integrated DNA Technology) with $11 \mu$ I DI water, $4 \mu \mathrm{l}$ 5X loading dye (cat. no. LC6678, Invitrogen), and $1 \mu$ I SYBR gold nucleic acid gel stain (cat. no. S11494, Invitrogen), while NCB samples were prepared by mixing the $16 \mu \mathrm{l}, 30 \mu \mathrm{M}$ NCB solution with $4 \mu \mathrm{IX}$ loading dye. After the gel was pre-run for $30 \mathrm{~min}$, each lane was loaded with $20 \mu \mathrm{NCB}$ samples or ladder and the gel was run at 50V, $5 \mathrm{~mA}$ for $800 \mathrm{~min}$. All gels were imaged on Syngene gel imager (with $365 \mathrm{~nm}$ excitation) to evaluate the mobility. As no SYBR gold dye was added to the NCB samples, the gel bands of NCBs showed their nature fluorescence colors (Supplementary Fig. S20).

\section{NCB elution from native PAGE gels}

NCB gel bands were extracted using a gel band cutter and collected in a $1.5 \mathrm{ml}$ Eppendorf tube, followed by smashing the gel into pieces with a plastic stick. For elution, $450 \mu \mathrm{l}$ of $20 \mathrm{mM} \mathrm{SPB} \mathrm{pH6.6}$ solution was added to each tube. The tube was shaken for $1 \mathrm{hr}$ and stored at room temperature overnight. The suspension was then filtered using a micro-centrifugal filter (cat. no. F2517-5, Thermo Scientific) at $8,000 \mathrm{~g}$ for $20 \mathrm{~min}$, followed by buffer exchange using the $0.5 \mathrm{ml}$ centrifugal filter described above (cat. no. UFC503024, MilliporeSigma) and $10 \mathrm{mM}$ ammonium acetate buffer (cat. no. AM9070G, Invitrogen).

\section{Sample preparation for native mass spectrometry}

Two gel-purified NCB samples (yPOT5 and rPOT5) were desalted and buffer exchanged into $10 \mathrm{mM}$ ammonium acetate using a spin column (Micro Bio-Spin ${ }^{\mathrm{TM}}$ P-6 Gel Columns, Bio-Rad). Octylamine was added to aliquots of NCB solution at a concentration of $0.1 \%(\mathrm{v} / \mathrm{v})$, to reduce the extensive metal cationic adduction that is commonly seen for ESI-MS analysis of oligonucleotides $>20 \mathrm{nt}^{40-43}$.

\section{Native mass spectrometry by direct infusion}

3-5 $\mu$ l of $5 \mu \mathrm{M}$ purified yPOT5 and rPOT5 NCB solutions were loaded into Au/Pd-coated nanospray borosilicate static tips (prepared in-house) for nano electrospray ionization (nESI). All direct infusion experiments were performed on a Thermo Scientific Q Exactive HF-X Hybrid Quadrupole-Orbitrap Mass Spectrometer. A spray voltage of $0.65-0.8 \mathrm{kV}$ and heated capillary temperature of $150{ }^{\circ} \mathrm{C}$ were used to ionize and desolvate the NCB complexes, facilitating their transmission into the gas-phase. Insource CID (80-110 eV) was also utilized to enhance transmission and reduce cationic adduction of the NCB complexes. MS1 spectra were collected at a resolution of 240K (@ m/z 200) and averaged over 100 scans (Supplementary Fig. S21).

\section{Acknowledgments}

This work was supported by the Welch Foundation to J.S.B. (F-1155) and H.-C.Y. (F-1833), the Texas 
4000 to H.-C.Y., National Institutes of Health to H.-C.Y. and I.J.F. (GM129617), and National Science Foundation to H.-C.Y. and J.S.B. (2029266).

\section{Author contributions}

Y.-A.K., C.J., Y.-A.C., J.T.P., I.J.F. and H.-C.Y. discussed and defined the project. I.J.F. and H.-C.Y. supervised the research. Y.-A.K., C.J., Y.-A.C., O.S.Z. and T.N.D. prepared the NCBs and carried out the imaging experiments. Y.-A.K., H.-C.K. prepared PCR samples for the MiSeq chip selection. Y.-A.K., O.S.Z., J.A.H and J.R.R. wrote the image alignment and analysis software. Y.-A.K. and O.S.Z. wrote flat-field correction script. Y.-A.K., O.S.Z., D.C.W. and S.H. made a comparison on machine learning algorithms and establish the predictive design workflow. Y.-A.K. and Y.-I.C performed fluorometry data analysis. J.N.W., S.W.S. and J.S.B. conducted direct infusion mass spectrum analysis. Y.-A.K., H.-C.Y., and I.J.F. wrote the paper with editorial assistance from all co-authors.

\section{Competing interests}

The authors declare no competing interests.

\section{Data availability}

CHAMP program is available at https://github.com/finkelsteinlab/champ. All other relevant raw/analyzed data are available from the corresponding authors upon reasonable request.

\section{Additional information}

Supplementary information. The online version contains supplementary material available at Nature Communications website. 


\section{References}

1 Kobayashi, H., Ogawa, M., Alford, R., Choyke, P. L. \& Urano, Y. New strategies for fluorescent probe design in medical diagnostic imaging. Chem Rev 110, 2620-2640, doi:10.1021/cr900263j (2010).

2 Chinen, A. B. et al. Nanoparticle Probes for the Detection of Cancer Biomarkers, Cells, and Tissues by Fluorescence. Chem Rev 115, 10530-10574, doi:10.1021/acs.chemrev.5b00321 (2015).

3 Rajendran, M. \& Ellington, A. D. In vitro selection of molecular beacons. Nucleic Acids Res 31, 5700-5713, doi:10.1093/nar/gkg764 (2003).

4 Yeh, H. C., Sharma, J., Han, J. J., Martinez, J. S. \& Werner, J. H. A DNA-silver nanocluster probe that fluoresces upon hybridization. Nano Letters 10, 3106-3110, doi:10.1021/nl101773c (2010).

5 Obliosca, J. M. et al. A complementary palette of NanoCluster Beacons. ACS Nano 8, 1015010160, doi:10.1021/nn505338e (2014).

6 Yeh, H.-C., Sharma, J., Han, J. J., Martinez, J. S. \& Werner, J. H. A beacon of light - a new molecular probe for homogeneous detection of nucleic acid targets. IEEE Nanotechnology Magazine 5, 28-33, doi:10.1109/mnano.2011.940951 (2011).

Ang, Y. S., Woon, W. W. E. \& Yung, L. Y. L. The role of spacer sequence in modulating turn-on fluorescence of DNA-templated silver nanoclusters. Nucleic Acids Research 46, 6974-6982, doi:10.1093/nar/gky521 (2018).

8 Obliosca, J. M., Liu, C. \& Yeh, H. C. Fluorescent silver nanoclusters as DNA probes. Nanoscale 5, 8443-8461, doi:10.1039/c3nr01601c (2013).

9 Schultz, D. et al. Evidence for rod-shaped DNA-stabilized silver nanocluster emitters. Adv Mater 25, 2797-2803, doi:10.1002/adma.201204624 (2013).

10 Copp, S. M. et al. Magic Numbers in DNA-Stabilized Fluorescent Silver Clusters Lead to Magic Colors. J Phys Chem Lett 5, 959-963, doi:10.1021/jz500146q (2014).

11 Petty, J. T. et al. A Segregated, Partially Oxidized, and Compact Ag10 Cluster within an Encapsulating DNA Host. J Am Chem Soc 138, 3469-3477, doi:10.1021/jacs.5b13124 (2016).

12 Huard, D. J. E. et al. Atomic Structure of a Fluorescent Ag8 Cluster Templated by a Multistranded DNA Scaffold. J Am Chem Soc 141, 11465-11470, doi:10.1021/jacs.8b12203 (2019).

13 Cerretani, C., Kanazawa, H., Vosch, T. \& Kondo, J. Crystal structure of a NIR-Emitting DNAStabilized Ag16 Nanocluster. Angew Chem Int Ed Engl, doi:10.1002/anie.201906766 (2019).

14 Yeh, H. C. et al. A fluorescence light-up Ag nanocluster probe that discriminates singlenucleotide variants by emission color. Journal of the American Chemical Society 134, 1155011558, doi:10.1021/ja3024737 (2012).

15 Copp, S. M., Bogdanov, P., Debord, M., Singh, A. \& Gwinn, E. Base motif recognition and design of DNA templates for fluorescent silver clusters by machine learning. Advanced Materials 26, 5839-5845, doi:10.1002/adma.201401402 (2014).

16 Juul, S. et al. NanoCluster Beacons as reporter probes in rolling circle enhanced enzyme activity detection. Nanoscale 7, 8332-8337, doi:10.1039/c5nr01705j (2015).

17 Chen, Y. A. et al. NanoCluster Beacons Enable Detection of a Single N(6)-Methyladenine. 
Journal of the American Chemical Society 137, 10476-10479, doi:10.1021/jacs.5b06038 (2015).

18 Petty, J. T. et al. A DNA-Encapsulated Silver Cluster and the Roles of Its Nucleobase Ligands. The Journal of Physical Chemistry C 122, 28382-28392, doi:10.1021/acs.jpcc.8b09414 (2018).

19 Chen, Y. A. et al. Improving NanoCluster Beacon performance by blocking the unlabeled NC probes. Chem Commun 55, 462-465, doi:10.1039/c8cc08291j (2019).

20 Zhang, J. et al. Hairpin DNA-templated silver nanoclusters as novel beacons in strand displacement amplification for microRNAs detection. Analytical Chemistry 88, 1294-1302, doi:10.1021/acs.analchem.5b03729 (2015).

21 Li, J. J., Zhong, X. Q., Zhang, H. Q., Le, X. C. \& Zhu, J. J. Binding-induced fluorescence turn-on assay using aptamer-functionalized silver nanocluster DNA probes. Analytical Chemistry 84, 5170-5174, doi:Doi 10.1021/Ac3006268 (2012).

22 Zhang, M., Guo, S. M., Li, Y. R., Zuo, P. \& Ye, B. C. A label-free fluorescent molecular beacon based on DNA-templated silver nanoclusters for detection of adenosine and adenosine deaminase. Chem Commun (Camb) 48, 5488-5490, doi:10.1039/c2cc31626a (2012).

23 Yin, J. et al. Label-free and turn-on aptamer strategy for cancer cells detection based on a DNAsilver nanocluster fluorescence upon recognition-induced hybridization. Analytical Chemistry 85 , 12011-12019, doi:10.1021/ac402989u (2013).

24 Richards, C. I. et al. Oligonucleotide-stabilized Ag nanocluster fluorophores. Journal of the American Chemical Society 130, 5038-5039, doi:10.1021/ja8005644 (2008).

25 Nutiu, R. et al. Direct measurement of DNA affinity landscapes on a high-throughput sequencing instrument. Nat Biotechnol 29, 659-664, doi:10.1038/nbt.1882 (2011).

26 Tome, J. M. et al. Comprehensive analysis of RNA-protein interactions by high-throughput sequencing-RNA affinity profiling. Nat Methods 11, 683-688, doi:10.1038/nmeth.2970 (2014).

27 Buenrostro, J. D. et al. Quantitative analysis of RNA-protein interactions on a massively parallel array reveals biophysical and evolutionary landscapes. Nat Biotechnol 32, 562-568, doi:10.1038/nbt.2880 (2014).

28 Jung, C. et al. Massively Parallel Biophysical Analysis of CRISPR-Cas Complexes on Next Generation Sequencing Chips. Cell 170, 35-47, doi:10.1016/j.cell.2017.05.044 (2017).

29 Layton, C. J., McMahon, P. L. \& Greenleaf, W. J. Large-Scale, Quantitative Protein Assays on a High-Throughput DNA Sequencing Chip. Mol Cell 73, 1075-1082 e1074, doi:10.1016/j.molcel.2019.02.019 (2019).

30 Hayward, S. L. et al. Ultraspecific and Amplification-Free Quantification of Mutant DNA by SingleMolecule Kinetic Fingerprinting. J Am Chem Soc 140, 11755-11762, doi:10.1021/jacs.8b06685 (2018).

31 Swasey, S. M., Leal, L. E., Lopez-Acevedo, O., Pavlovich, J. \& Gwinn, E. G. Silver (I) as DNA glue: $\mathrm{Ag}(+)$-mediated guanine pairing revealed by removing Watson-Crick constraints. Sci Rep 5, 10163, doi:10.1038/srep10163 (2015).

32 Kondo, J. et al. A metallo-DNA nanowire with uninterrupted one-dimensional silver array. Nat Chem 9, 956-960, doi:10.1038/nchem.2808 (2017).

33 Sharma, J., Yeh, H. C., Yoo, H., Werner, J. H. \& Martinez, J. S. A complementary palette of 
fluorescent silver nanoclusters. Chemical Communcations 46, 3280-3282, doi:10.1039/b927268b (2010).

34 Hsin-Chih, Y., Jaswinder, S., Hyojong, Y., Jennifer, S. M. \& James, H. W. Photophysical characterization of fluorescent metal nanoclusters synthesized using oligonucleotides, proteins and small molecule ligands. Proc. SPIE 7576, doi:10.1117/12.842192 (2010).

35 Blevins, M. S. et al. Footprints of Nanoscale DNA-Silver Cluster Chromophores via ActivatedElectron Photodetachment Mass Spectrometry. ACS Nano 13, 14070-14079, doi:10.1021/acsnano.9b06470 (2019).

36 Petty, J. T., Ganguly, M., Rankine, I. J., Chevrier, D. M. \& Zhang, P. A DNA-Encapsulated and Fluorescent Ag106+ Cluster with a Distinct Metal-Like Core. The Journal of Physical Chemistry C 121, 14936-14945, doi:10.1021/acs.jpcc.7b04506 (2017).

37 Cerretani, C., Kondo, J. \& Vosch, T. Removal of the A10 adenosine in a DNA-stabilized Ag16 nanocluster. RSC Advances 10, 23854-23860, doi:10.1039/d0ra02672g (2020).

38 O'Neill, P. R., Velazquez, L. R., Dunn, D. G., Gwinn, E. G. \& Fygenson, D. K. Hairpins with PolyC Loops Stabilize Four Types of Fluorescent Agn:DNA. The Journal of Physical Chemistry C 113, 4229-4233, doi:10.1021/jp809274m (2009).

39 Schultz, D. \& Gwinn, E. G. Silver atom and strand numbers in fluorescent and dark Ag:DNAs. Chem Commun (Camb) 48, 5748-5750, doi:10.1039/c2cc17675k (2012).

40 McGinnis, A. C., Grubb, E. C. \& Bartlett, M. G. Systematic optimization of ion-pairing agents and hexafluoroisopropanol for enhanced electrospray ionization mass spectrometry of oligonucleotides. Rapid Commun Mass Spectrom 27, 2655-2664, doi:10.1002/rcm.6733 (2013).

41 Scalabrin, M., Palumbo, M. \& Richter, S. N. Highly Improved Electrospray Ionization-Mass Spectrometry Detection of G-Quadruplex-Folded Oligonucleotides and Their Complexes with Small Molecules. Anal Chem 89, 8632-8637, doi:10.1021/acs.analchem.7b01282 (2017).

Sutton, J. M. \& Bartlett, M. G. Modeling cationic adduction of oligonucleotides using electrospray desorption ionization. Rapid Commun Mass Spectrom 34, e8696, doi:10.1002/rcm.8696 (2020).

43 Sutton, J. M., El Zahar, N. M. \& Bartlett, M. G. Oligonucleotide Anion Adduct Formation Using Negative Ion Electrospray lon-Mobility Mass Spectrometry. J Am Soc Mass Spectrom 32, 497508, doi:10.1021/jasms.0c00380 (2021).

44 Cerretani, C., Kondo, J. \& Vosch, T. Mutation of position 5 as a crystal engineering tool for a NIR-emitting DNA-stabilized Ag16 nanocluster. CrystEngComm 22, 8136-8141, doi:10.1039/d0ce01225d (2020).

45 Copp, S. M. et al. Fluorescence Color by Data-Driven Design of Genomic Silver Clusters. ACS Nano, doi:10.1021/acsnano.8b03404 (2018).

46 Copp, S. M., Swasey, S. M., Gorovits, A., Bogdanov, P. \& Gwinn, E. G. General Approach for Machine Learning-Aided Design of DNA-Stabilized Silver Clusters. Chemistry of Materials 32, 430-437, doi:10.1021/acs.chemmater.9b04040 (2019).

47 Vens, C., Rosso, M. N. \& Danchin, E. G. Identifying discriminative classification-based motifs in biological sequences. Bioinformatics 27, 1231-1238, doi:10.1093/bioinformatics/btr110 (2011). Hall, M. et al. The WEKA data mining software. ACM SIGKDD Explorations Newsletter 11, 10- 
18, doi:10.1145/1656274.1656278 (2009).

49 Ristad, E. S. \& Yianilos, P. N. Learning string-edit distance. IEEE Transactions on Pattern Analysis and Machine Intelligence 20, 522-532, doi:10.1109/34.682181 (1998).

50 Del Bonis-O'Donnell, J. T. et al. DNA-Stabilized Silver Nanoclusters as Specific, Ratiometric Fluorescent Dopamine Sensors. ACS Chem Neurosci 9, 849-857, doi:10.1021/acschemneuro.7b00444 (2018).

51 Chen, W. Y., Lan, G. Y. \& Chang, H. T. Use of fluorescent DNA-templated gold/silver nanoclusters for the detection of sulfide ions. Anal Chem 83, 9450-9455, doi:10.1021/ac202162u (2011).

52 Jia, X. et al. DNA-hosted copper nanoclusters for fluorescent identification of single nucleotide polymorphisms. ACS Nano 6, 3311-3317, doi:10.1021/nn3002455 (2012).

53 Chen, J., Ji, X., Tinnefeld, P. \& He, Z. Multifunctional Dumbbell-Shaped DNA-Templated Selective Formation of Fluorescent Silver Nanoclusters or Copper Nanoparticles for Sensitive Detection of Biomolecules. ACS Appl Mater Interfaces 8, 1786-1794, doi:10.1021/acsami.5b09678 (2016).

54 Edelstein, A. D. et al. Advanced methods of microscope control using muManager software. J Biol Methods 1, doi:10.14440/jbm.2014.36 (2014).

55 Hall, M. A.; Smith, L. A. Practical Feature Subset Selection for Machine Learning. In Proceedings of the 21st Australasian Computer Science Conference ACSC'98; Springer, Berlin, Heidelberg: Perth, 1998; pp 181-191.

56 Hall, M. A. \& Smith, L. A. Vol. Volume 20 No 1 181-191 (Springer, Conference held at Perth, 1998). 
a

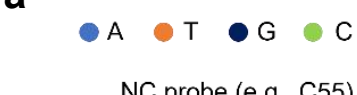

NC probe (e.g., C55) activator (e.g., G15) b



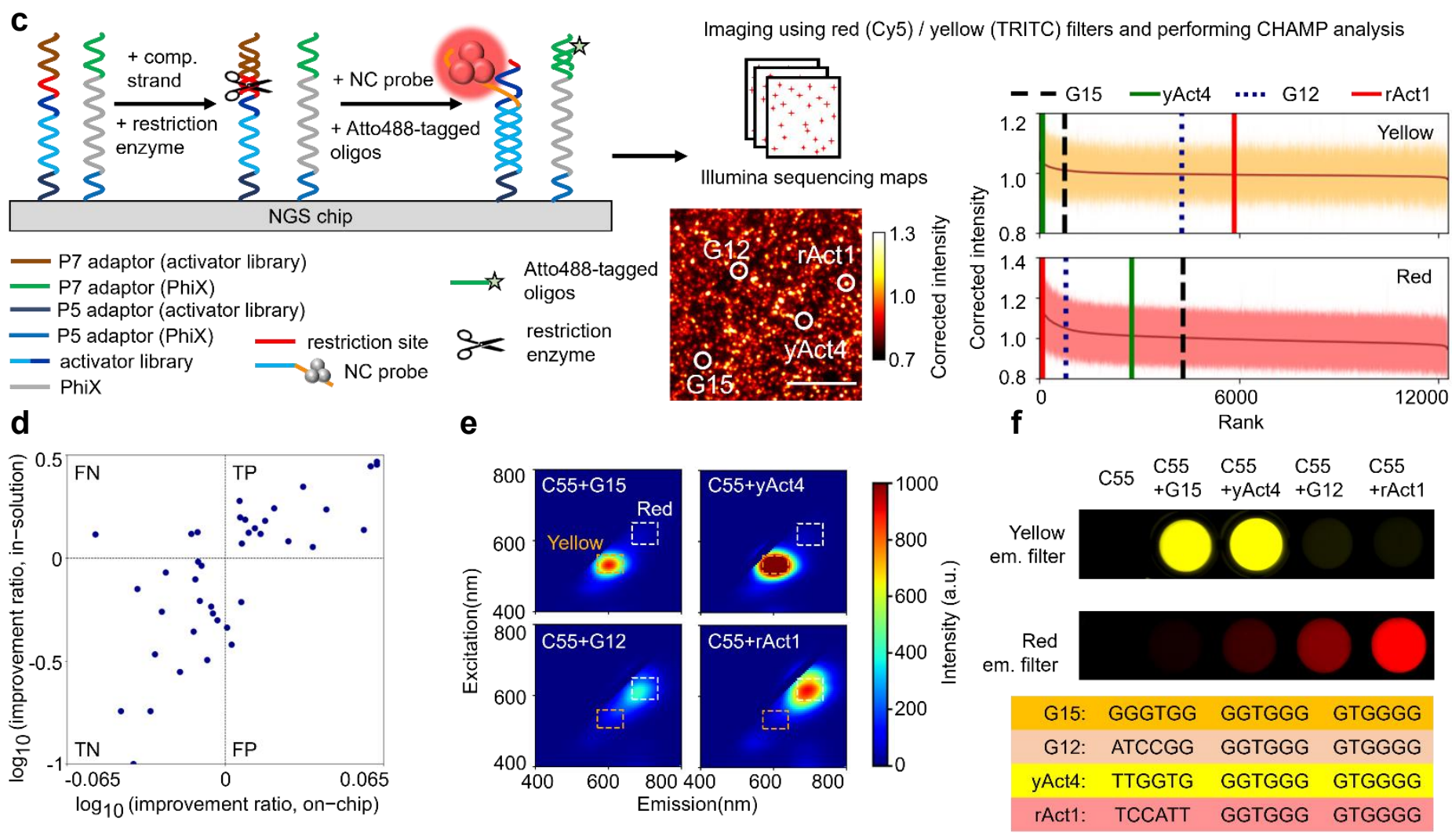

Fig. 1 Massively parallel selection of NanoCluster Beacons (NCBs) using MiSeq chips. a The interactions between a silver nanocluster (AgNC, left) and a proximal guanine-rich activator (middle) activate the fluorescence of AgNC by hundreds to thousands fold, creating an activated NCB (right). Here a common C55 nanocluster (NC) probe is used for NCB selection and optimization. G15 is the canonical activator for yellow NCB. b C55 NC probe before and after activation by G15 activator, under UV excitation (365 nm). c Workflow of our high-throughput NCB selection on a next-generation sequencing chip (NGS; MiSeq, Illumina). After sequencing a library of activators $(>12,000)$ on the MiSeq chip, unwanted sequence above the activator was cleaved by a restriction enzyme. The Atto488-tagged fiducial marker probes and the C55 probes were then injected into the chip to hybridize with the PhiX markers and the library, and imaged sequentially under an epifluorescence microscope. A custom bioinformatics and imaging processing pipeline was employed to identify activator sequence behind each activated NCB spot. After ranking all activators based on their median activation brightness, we could clearly differentiate strong activators from weak ones in the yellow (Ex/Em: 535/50, 605/70 $\mathrm{nm}$ ) and red (Ex/Em: 620/60, 700/75 nm) emission channels. Here G15 and G12 were the standards (the known best) for the yellow and red NCB comparisons, respectively. Both G15 and G12 ranking 800 among the yellow and red NCBs in this library (library_1). $\mathbf{d}$ Twenty top-ranked and twenty bottom-ranked activators were further investigated in test tubes using traditional florometry. The MiSeq results were $85 \%$ accurate in both true positive (TP) and true negative (TN) selections. Definition of the improvement ratio can be found in the methods. e $2 \mathrm{D}$ spectra of the four representative NCBs in the yellow (orange dashed box) and red (white dashed box) emission channels. Through florometry characterization, we found yAct4 2.03-fold brighter than G15 and rAct1 2.94-fold 
brighter than G12. Intensities were calculated based on a volumetric integral shown in Supplementary Fig. S2. f. Plate-reader images acquired using yellow (top) and red (bottom) excitation/emission filter sets, and the sequences of the four representative bright NCBs. 
a

\begin{tabular}{|c|l|l|l|}
\hline Canonical & \multicolumn{3}{|c|}{ 5' - GGGTGGGGTGGGGTGGGG - 3' } \\
\hline $\begin{array}{c}\text { Segment_1, } \\
\text { library_1 }\end{array}$ & \multirow{2}{*}{ NNNNNNGGTGGGGTGGGG } & Segment_11 & NNNTGGGGTGGGGTGGGG \\
\cline { 3 - 4 } & Segment_12 & GGGNNNGGTGGGGTGGGG \\
\hline $\begin{array}{c}\text { Segment_2, } \\
\text { library_1 }\end{array}$ & \multirow{2}{*}{ GGGTGGNNNNNNGTGGGG } & Segment_21 & GGGTGGNNNGGGGTGGGG \\
\cline { 3 - 4 } & Segment_22 & GGGTGGGGTNNNGTGGGG \\
\hline $\begin{array}{c}\text { Segment_3, } \\
\text { library_1 }\end{array}$ & \multirow{2}{*}{ GGGTGGGGTGGGNNNNNN } & Segment_31 & GGGTGGGGTGGGNNNGGG \\
\cline { 3 - 4 } & Segment_32 & GGGTGGGGTGGGGTGNNN \\
\hline
\end{tabular}

b $\quad \begin{array}{ccc}\text { Segment_1 } & \text { Segment_2 } & \text { Segment_3 } \\ \text { (zipper, pos. 1-6) } & (\text { bag, pos. 7-12) } & \text { (pos. 13-18) }\end{array}$

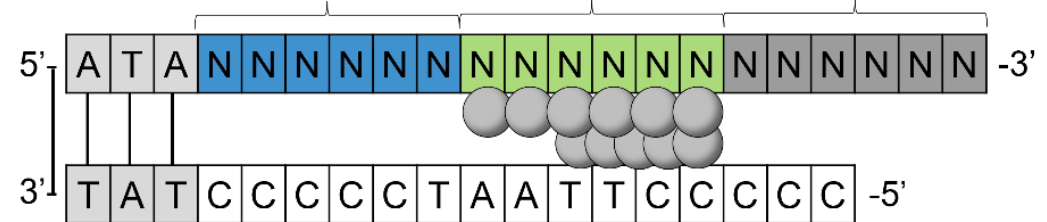
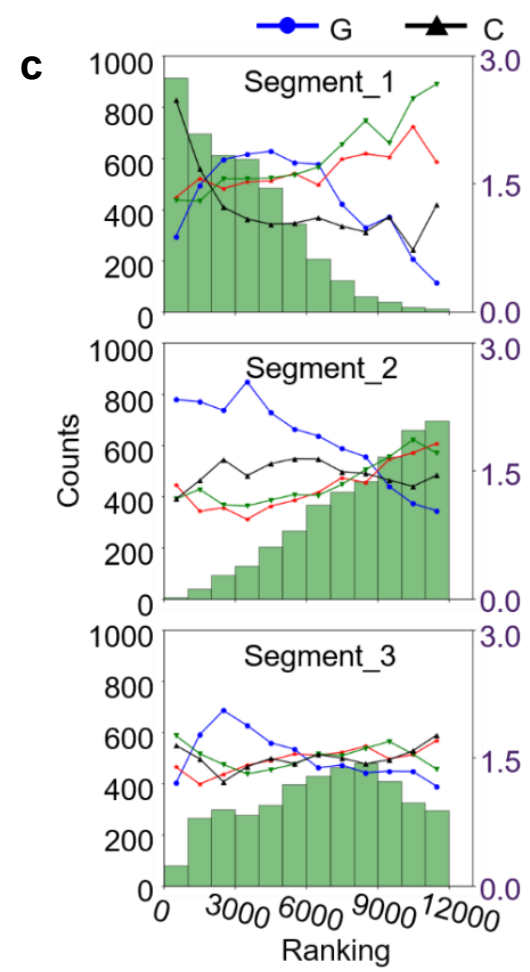

$\star A \rightarrow T$

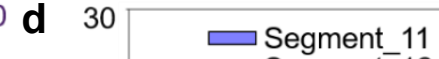
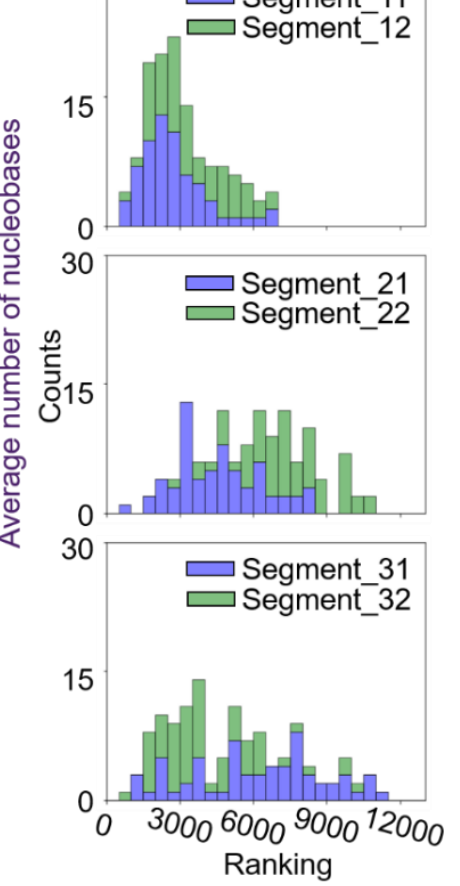

Fig. 2 Influence of activator mutations on red NCB brightness. a In library_1, the 18-nt-long canonical activator $\mathrm{G} 15$ was divided into three 6 -nt-long segments and each segment was separately randomized, creating a library with total 12,286 activators. b Schematic of NCB construct and definition of nucleobase positions in the activator. c Histograms of the brightness rankings corresponding to the mutated segments and the average numbers of the 4 nucleobases in the mutated segments. The library_1 results clearly indicated that, to make a bright NCB, segment_2 (the middle 6 nucleobases, positions 7-12) prefers the canonical G-rich sequence, as randomizing segment_2 (while keeping segment_1 and_3 canonical) leads to many low-ranking NCBs in both emission channels. Each histogram contained 4,096 activators. d The 6-segment interrogation further demonstrated that segment_22 (positions 10-12) is more important than segment_21 (positions 7-9) in creating bright red NCBs. 
a

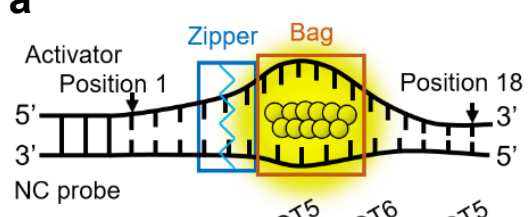

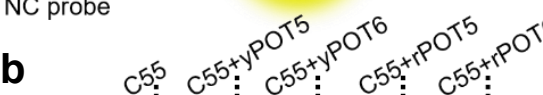

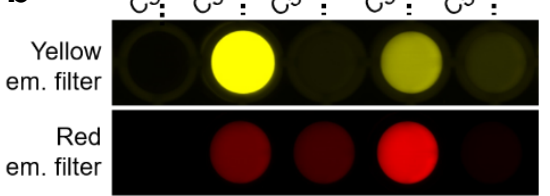

กㅇ $\square$ Yellow channel $\square$ Red channel

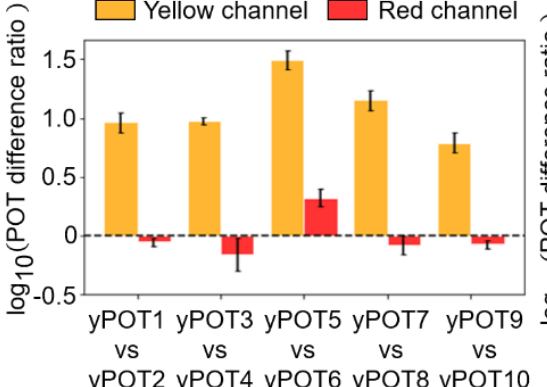

уРОT2 уРОТ4 уРОТ6 уРОТ8 уРОТ10
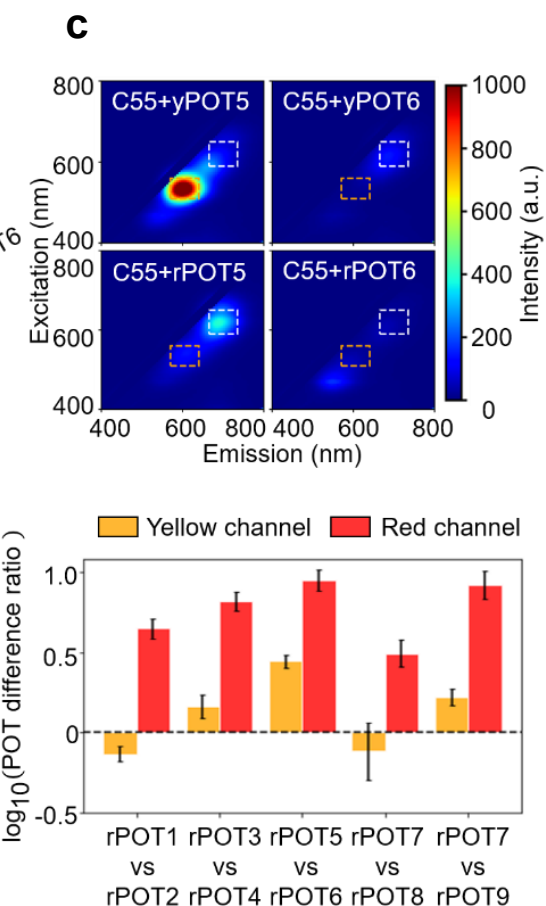

d
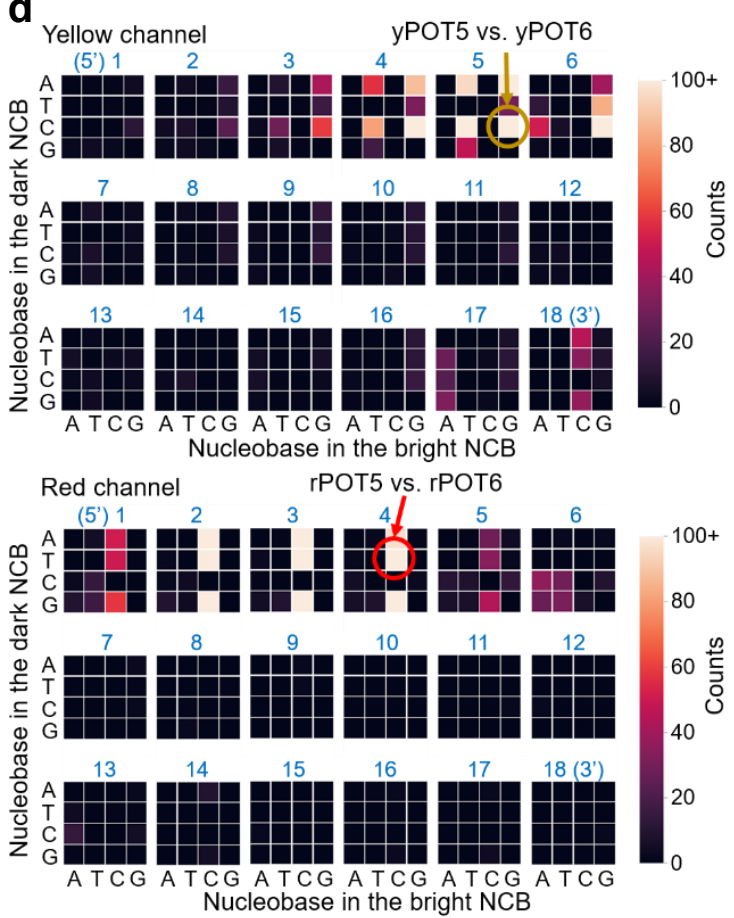

Fig. 3 Substitution hotspots to generate polar opposite twins (POTs) revealed by MiSeq chip selection. a Schematic of the zipper bag model. The blue box represents the "zipper" location (e.g., positions 4-6 for yellow POTs) and the orange box represents the "bag" location (i.e., the critical zone at positions 7-12). When the zipper does not seal well, the bag is leaky, thus leading to a low chemical yield and dimmer NCB. $\mathbf{b}$ Plate-reader images acquired using yellow (top) and red (bottom) excitation/emission filter sets and the sequences of the representative POTs. Large differences in fluorescence enhancement ratios were seen in these twin NCBs (C55+yPOT5 vs. C55+yPOT6 for yellow channel, and C55+rPOT5 vs. C55+rPOT6 for red channel), making them POTs. c 2D spectra of the representative POTs in the yellow (orange dashed box) and red (white dashed box) emission channels. d Heat maps of the top 2,000 twin NCB pairs in library_1. Here the x-axis and the yaxis represent bright to dark conversion in these twin NCBs. These heat maps clearly indicated that the nucleobases in positions 4-6 are critical for creating yellow POTs, while the positions 2-4 are critical for creating red POTs. e The POT difference ratios of five representative yellow (left) and red (right) pairs of POTs in the two emission channels. Through fluorometry characterization, the yPOT5-yPOT6 pair and the rPOT5-rPOT6 pair were identified as the most extreme yellow and red POTs, respectively, reaching POT difference ratios as high as 31 and 9. Definition of the POT difference ratio can be found in the methods. Error bars: mean \pm s.d. in logarithmic scale, with 3 repeats for each pair of POTs. 
a

\begin{tabular}{|c|}
\hline \multicolumn{2}{|c|}{ Ranking list (yellow) } \\
\hline Ranking list (red) \\
\hline TCCACTGGTGGGGTGGGG \\
GGGTGGGGTGGGACTCAG \\
GGGTGGCCGGTTGTGGGG \\
\hline$\ldots$ \\
\hline TTGATAGGTGGGGTGGGG \\
GGGTGGCTGAACGTGGGG \\
GGGTGGGGTGGGCCCCCA \\
\hline
\end{tabular}

b

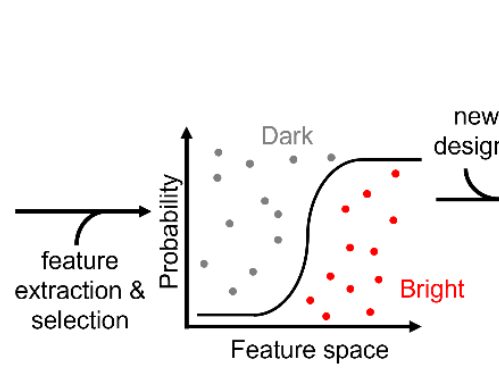

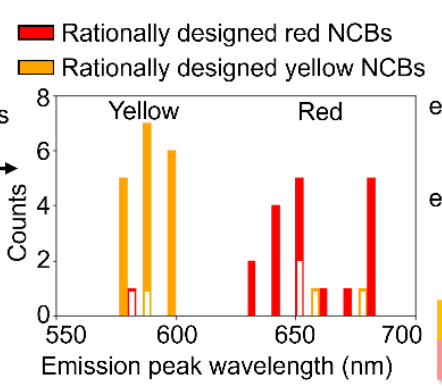

C

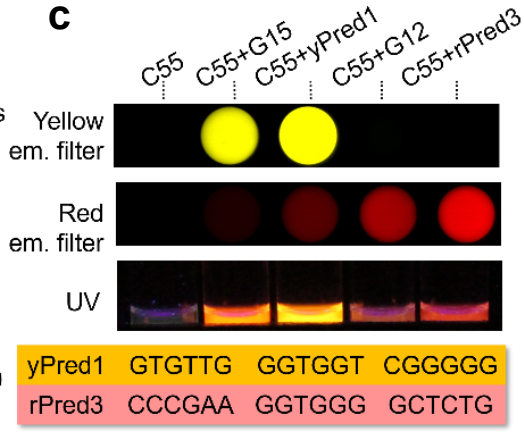

Fig. 4 Predictive design of bright yellow and red NCBs based on machine learning results. a From the chip selection results, we labeled the top 30\% NCBs as 'bright' class and the bottom $30 \%$ as 'dark' class. The sequence features of these selected NCBs were then extracted by $\mathrm{MERCl}$ and selected by Weka. The resulting feature vectors thus defined the location of individual activators in the high-dimensional space. Several machine learning models were tested for activator classification, among which the logistic regression had the best performance. b Forty new activators were rationally designed and tested by fluorometry. Three out of the 20 rationally designed red NCBs actually showed either low emission or yellow emission (85\% test-tube validation accuracy), while three of the 20 rationally designed yellow NCBs showed low emission of red emission (also $85 \%$ test-tube validation accuracy). Empty boxes represent the failed designs with low emission. c Plate-reader images acquired using yellow (top) and red (bottom) excitation/emission filter sets, and the activator sequences of the two successfully predicted NCBs and one failed prediction. 